Numerical Mathematics

Elsevier Editorial System(tm) for Applied

Manuscript Draft

Manuscript Number:

Title: Structure preserving preconditioners for image deblurring

Article Type: SI:NETNA 2015

Keywords: Regularization; preconditioning; Toeplitz matrices

Corresponding Author: Prof. Claudio Estatico,

Corresponding Author's Institution: University of Genova

First Author: Pietro Dell'Acqua

Order of Authors: Pietro Dell'Acqua; Marco Donatelli; Claudio Estatico; Mariarosa Mazza

Abstract: Regularizing preconditioners for accelerating the convergence of iterative regularization methods

without spoiling the quality of the approximated solution

have been extensively investigated in the last twenty years.

Several strategies have been proposed for defining proper

preconditioners.

Usually, in methods for image restoration, the structure of the

preconditioner is chosen Block Circulant with Circulant Blocks (BCCB)

because it can be efficiently exploited by Fast Fourier Transform (FFT).

Nevertheless, for ill-conditioned problems, it is well known that BCCB

preconditioners cannot provide a strong clustering of the eigenvalues.

Moreover, in order to get an effective preconditioner, it is crucial to preserve the structure of the coefficient matrix.

The structure of such matrix, in case of image deblurring problem, depends on the boundary conditions imposed on the imaging model.

Therefore we propose a technique to construct a preconditioner which has the same structure of the blurring matrix related to the restoration problem at hand.

The construction of our preconditioner requires two FFTs like the BCCB preconditioner.

The presented preconditioning strategy represents a generalization and an improvement with respect of both circulant and structured preconditioning available in the literature.

The technique is further extended to provide a non-stationary preconditioning in the same spirit of a recent proposal for BCCB matrices.

Some numerical results show the importance of preserving the matrix structure from the point of view of both restoration quality and robustness of the regularization parameter. 


\title{
Structure preserving preconditioners for image deblurring
}

\author{
Pietro Dell' Acqua ${ }^{\mathrm{a}}$, Marco Donatelli ${ }^{\mathrm{a}}$, Claudio Estatico ${ }^{\mathrm{b}, *}$, Mariarosa Mazza $^{\mathrm{a}}$ \\ ${ }^{a}$ Department of Science and High Technology, University of Insubria, via Valleggio 11, 22100, Como, Italy \\ ${ }^{b}$ Department of Mathematics, University of Genova, Via Dodecaneso, 35 - 16146 Genova, Italy
}

\begin{abstract}
Regularizing preconditioners for accelerating the convergence of iterative regularization methods without spoiling the quality of the approximated solution have been extensively investigated in the last twenty years. Several strategies have been proposed for defining proper preconditioners. Usually, in methods for image restoration, the structure of the preconditioner is chosen Block Circulant with Circulant Blocks (BCCB) because it can be efficiently exploited by Fast Fourier Transform (FFT). Nevertheless, for ill-conditioned problems, it is well known that BCCB preconditioners cannot provide a strong clustering of the eigenvalues. Moreover, in order to get an effective preconditioner, it is crucial to preserve the structure of the coefficient matrix. The structure of such matrix, in case of image deblurring problem, depends on the boundary conditions imposed on the imaging model. Therefore we propose a technique to construct a preconditioner which has the same structure of the blurring matrix related to the restoration problem at hand. The construction of our preconditioner requires two FFTs like the BCCB preconditioner. The presented preconditioning strategy represents a generalization and an improvement with respect of both circulant and structured preconditioning available in the literature. The technique is further extended to provide a non-stationary preconditioning in the same spirit of a recent proposal for BCCB matrices. Some numerical results show the importance of preserving the matrix structure from the point of view of both restoration quality and robustness of the regularization parameter.
\end{abstract}

Keywords: Regularization, preconditioning, Toeplitz matrices.

\section{Introduction}

Let us consider the linear system

$$
A x=b,
$$

where $A \in \mathbb{R}^{n \times n}$ and $x, b \in \mathbb{R}^{n}$. In the image deblurring context, the matrix $A$ represents the blurring operator created according to the Point Spread Function (PSF) and the Boundary Conditions (BCs), $x$ is an approximation of the true image $\bar{x} \in \mathbb{R}^{n}$ of an unknown object and $b$ is the detected image affected by blur and corrupted by a noise $\eta \in \mathbb{R}^{n}$, i.e. $b=A \bar{x}+\eta$. Image deblurring consists in computing an approximation of the true image $\bar{x}$ by means of an appropriate solution of (1). Since the singular values of $A$ gradually approach zero without a significant gap, $A$ is very ill-conditioned and may be singular. Linear systems of equations with a matrix of this kind are commonly referred as linear discrete ill-posed problems and require regularization [1].

The special structure of the square blurring matrix $A$ depends on the properties of the basic blurring model, i.e. the PSF and the BCs. In this work we assume that the blurring model is space-invariant and that the BCs are defined as affine relations between the unknowns inside the Field Of View (FOV). BCs try to capture and to include into the deblurring model the unknown behaviour of the signal outside the FOV in which the detection is made [2]. Indeed, the information inside the FOV contained in the detected image $b$ is incomplete and does not allow to restore the true image even in the (unrealistic) noiseless case. Among the BCs present in the literature, we consider the following ones.

In the Zero (Dirichelet) BCs model, the image outside the FOV is supposed to be null, i.e. zero pixel-valued. In the Periodic BCs model, the image inside the FOV is periodically repeated outside the FOV. In the Reflective BCs model the image inside the FOV is reflected outside the FOV, as there were a vertical mirror along each edge. That way, the pixel values across the boundary are extended so that the continuity of the image is preserved at the boundary. In the AntiReflective BCs model the image inside the FOV is anti-reflected outside the FOV. That way, the pixel values across the

\footnotetext{
*corresponding author

Email addresses: pietro.dellacqua@gmail.com (Pietro Dell'Acqua), marco.donatelli@uninsubria.it (Marco Donatelli), estatico@dima.unige.it (Claudio Estatico), mariarosa.mazza@uninsubria.it (Mariarosa Mazza)
} 
boundary are extended so that the continuity of the image and the continuity of the normal derivatives are both preserved at the boundary. Moving along the BCs listed here, the structure of the blurring matrix $A$ changes, becoming more involved. As we will see in the rest of the paper, by using Zero BCs we get a Block Toeplitz with Toeplitz Blocks (BTTB) matrix, by using Periodic BCs we get a Block Circulant with Circulant Blocks (BCCB) matrix, while Reflective and Anti-Reflective $\mathrm{BCs}$ give rise to more complex matrix structures [15].

The Zero BCs can be useful for some applications in astronomy, where an empty dark space surrounds a well located object. On the other hand, it gives rise to high ringing effects close to the boundary of the restored image in other classical imaging applications, where the background is not uniform. Periodic BCs are computational favourable since the matrix $A$ can be easily diagonalized by Fast Fourier Transform (FFT). The other two BCs are able to reduce these ringing effects; in particular, Anti-Reflective BCs can be considered the most precise, since no discontinuity in both the image and its normal derivatives is artificially added by the BCs imposed. In all these cases, the matrix-vector product can be done in $O(n \log n)$ by FFT, using a proper pad of the vector in agreement with the BCs imposed and then performing a circulant convolution of double size. In some cases, Reflective and Anti-Reflective BCs are even cheaper, since they require only real operations instead of complex ones [3] without needing any padding.

Regardless of the imposed BCs, if we require to deal with positive (semi-)definite matrices, instead of the system (1), we can solve the system of the normal equations

$$
A^{H} A x=A^{H} b,
$$

where $A^{H}$ is the conjugate transpose of $A$. This choice allows us to use many iterative methods, such as the Conjugate Gradient (CG) and its generalizations. Moreover, all iterative methods, when are applied to the normal equations (2), become more stable, i.e. less sensitive with respect to data noise. Unfortunately, in solving (2) instead of (1), the rate of convergence slows down. In this respect, the conventional technique to speed up the convergence is to consider the preconditioned system

$$
D A^{H} A x=D A^{H} b,
$$

where $D$ is the so-called preconditioner, whose role is to suitably approximate the (generalized) inverse of the normal matrix $A^{H} A$ [4]. With a careful look, we can say that the classical preconditioning scenario seems to be quite curious, since the preconditioner $D$ has to speed up the slowing down produced by $A^{H}$. On these grounds, in [5] we proposed a new technique that uses a single preconditioning operator directly applied to the original system (1). The new preconditioner, called as reblurring matrix $Z$, according to the terminology introduced in [6], leads to the new preconditioned system

$$
Z A x=Z b .
$$

As pointed out in [5], the aim of the preconditioner $Z$ is to allow iterative methods to become more stable (as well as usually obtained through the normal equations involving $A^{H}$ ) without slowing the convergence (so that no subsequent accelerating operator $D$ is needed), especially in the so-called signal space, i.e. the subspace less sensitive to the data noise. We introduced two different mathematical techniques to build the preconditioner $Z$. The first is based on a coarse version of the PSF, the second to a regularized (i.e. filtered) approximation of the conjugate of the inverse of the eigenvalues of the matrix $A$, approximated by using the generating function of the system matrix $A$. In both cases, in [5] we apply these techniques to compute a BCCB matrix $Z$, regardless of the BCs used in the model to define the system matrix $A$. Basically, the two techniques of [5] allow the preconditioner $Z$ to inherit some information on the spectrum of the system matrix $A$, but no information about its structure is used.

Moreover, it is well known by seminal results of [7], that a BCCB preconditioner of a BTTB matrix $A$ cannot be optimal even for an optimal (i.e. "almost exact") approximation of its generating function (and this yields that the BCCB preconditioner $Z$ used in [5] will never be optimal). On the other hand, a BTTB preconditioner of a BTTB matrix $A$ (i.e. a preconditioner with the same structure of the system matrix), is optimal even when the spectrum of the preconditioner is a poor approximation of the spectrum of the system matrix [8] (in particular, it is enough that all the zeros of the generating function are exactly located with the same multiplicity, regardless the other values of the generating function).

Based on all of these considerations, in this paper we improve the reblurring technique based on the approximation of the conjugate of the eigenvalues of the matrix $A$, by defining a class of preconditioners $Z$ endowed with the same structure of the system matrix $A$. We call this strategy as structure preserving reblurring preconditioners. At the best of our knowledge, the only papers in which the structure of $A$ is preserved in the preconditioner are $[9,10,11]$ for Zero, Reflective and Anti-Reflective BCs, respectively. In all three cases, the results reported heavily depend on the symmetry properties of the PSF. In particular, [9] takes into account a symmetric PSF and analyses preconditioned MR-II (a variant of the minimal residual method sometime also called conjugate residual), building the preconditioner by the well-known circulant padding of Toeplitz matrices. On the other hand, both [10,11] show that the optimal (in the sense of Frobenius norm) preconditioner in a proper algebra diagonalized by a fixed real transform is associated with the symmetrized version 
of the original PSF. Such preconditioning technique works well when the PSF is close to be symmetric, while has poor performance for strongly non-symmetric PSFs.

In this paper we propose a general preconditioning technique which can be used for any type of PSF and BCs. Our proposal belongs to the class of reblurring preconditioning (4), which was shown in [5] to be superior of the standard preconditioning (3) studied in [4], and it does not require any padding. We introduce the idea, by giving a simple and complete scheme to compute an approximated and regularized version of the inverse of the generating function of the system matrix $A$, which requires only the application of two FFTs in $O(n \log n)$. Moreover, in the special case of Zero $\mathrm{BCs}$, some relationships with the preconditioning strategy proposed in [9] are studied in detail.

All regularizing preconditioners require the estimation of a further filtering of the spectrum. A possible alternative is a non-stationary sequence of thresholding parameters as proposed in [12]. Since the proposal in [12] can be interpreted as a non-stationary variant of a specific reblurring preconditioner among those investigated in [5], in this paper we study also a possible non-stationary generalization of our structured preconditioner. In particular, similarly to the strategy adopted in [13] for low-rank approximations, we estimate the thresholding parameter at every iteration by resorting to a BCCB approximation which allows a simple and cheap computational solution of the corresponding non-linear problem. Finally, according to our numerical tests, we note that the use of a structured preconditioner is useful both to obtain a better restoration and a more robust choice of the regularization parameters with respect to classical BCCB preconditioners.

This paper is organized as follows. Section 2 reviews the structure of the blurring matrix in the case of space-invariant blur. Our structured preconditioner is introduced in Section 3, where similarities and differences with the approach in [9] are studied in detail in the case of symmetric Toeplitz matrices and Tikhonov filter. A non-stationary version of the structured preconditioner, inspired by the proposal in [12], is described in Section 4. Section 5 collects some numerical results and related comments. Finally, Section 6 is devoted to conclusions and discussion of future works.

\section{Structure of the blurring matrix}

The constitution of the blurring matrix $A$ is based on two ingredients: the PSF and the BCs enforced in the discretization. As already sketched in the Introduction, the latter choice gives rise to different types of structured matrices. For the sake of notational simplicity, we consider a square PSF $H \in \mathbb{R}^{n \times n}$. We suppose that the position of the PSF centre is known. Thus, $H$ can be depicted in this way

$$
H=\left(\begin{array}{ccccccc}
h_{-m_{1},-m_{2}} & \cdots & & h_{-m_{1}, 0} & & \cdots & h_{-m_{1}, n_{2}} \\
& & & & & & \\
\vdots & \ddots & & \vdots & & & \vdots \\
& & h_{-1,-1} & h_{-1,0} & h_{-1,1} & & \\
h_{0,-m_{2}} & \cdots & h_{0,-1} & h_{0,0} & h_{0,1} & \cdots & h_{0, n_{2}} \\
& & h_{1,-1} & h_{1,0} & h_{1,1} & & \\
\vdots & & & \vdots & & \ddots & \vdots \\
h_{n_{1},-m_{2}} & \cdots & & h_{n_{1}, 0} & & \cdots & h_{n_{1}, n_{2}}
\end{array}\right)_{n \times n},
$$

where $h_{0,0}$ is the central coefficient and $m_{1}+n_{1}+1=m_{2}+n_{2}+1=n$.

Given the pixels $h_{j_{1}, j_{2}}$ of the PSF, it is possible to associate the so-called generating function $f: \mathbb{R}^{2} \rightarrow \mathbb{C}$ as follows

$$
f\left(x_{1}, x_{2}\right)=\sum_{j_{1}=-m_{1}}^{n_{1}} \sum_{j_{2}=-m_{2}}^{n_{2}} h_{j_{1}, j_{2}} \mathrm{e}^{\hat{\imath}\left(j_{1} x_{1}+j_{2} x_{2}\right)}=\sum_{j_{1}, j_{2}=-n+1}^{n-1} h_{j_{1}, j_{2}} \mathrm{e}^{\hat{\imath}\left(j_{1} x_{1}+j_{2} x_{2}\right)},
$$

where $\hat{\imath}^{2}=-1$ and with the assumption that $h_{j_{1}, j_{2}}=0$ if the corresponding pixel is not detected, i.e. if the element $\left(h_{j_{1}, j_{2}}\right)$ does not belong to $H$ [14]. Note that $h_{j_{1}, j_{2}}$ are the Fourier coefficients of $f \in \Pi_{n-1}$, where $\Pi_{k}=\operatorname{span}\left\{\mathrm{e}^{\hat{\imath}\left(j_{1} x_{1}+j_{2} x_{2}\right)}, j_{1}, j_{2}=\right.$ $-k+1, \ldots, k-1\}$, so that the generating function $f$ contains the same information of $H$.

The most simple boundary conditions are Zero BCs, which assume that the signal is null outside the FOV. This is a good choice when we deal with images having a black background, occurring for instance in astronomical applications. In the other cases, such BCs usually give rise to heavy ringing phenomena near the boundaries. The resulting blurring 
matrix is a BTTB matrix. We will use the symbol $\mathcal{T}$ to denote this class of matrices. In detail, we have

$$
\mathcal{T}_{n}(f)=\left(\begin{array}{cccc}
T_{0} & T_{-1} & \cdots & T_{-n+1} \\
T_{1} & \ddots & \ddots & \vdots \\
\vdots & \ddots & \ddots & T_{-1} \\
T_{n-1} & \cdots & T_{1} & T_{0}
\end{array}\right)_{n^{2} \times n^{2}} \quad, \quad \text { with } \quad T_{k}=\left(\begin{array}{cccc}
h_{k, 0} & h_{k,-1} & \cdots & h_{k,-n+1} \\
h_{k, 1} & \ddots & \ddots & \vdots \\
\vdots & \ddots & \ddots & h_{k,-1} \\
h_{k, n-1} & \cdots & h_{k, 1} & h_{k, 0}
\end{array}\right)_{n \times n},
$$

for $k=-n+1, \ldots, n-1$. We highlight that we do not have fast trigonometric transforms for diagonalizing BTTB matrices. This represents an important drawback in using Zero BCs with filtering methods like classical Tikhonov. On the contrary, for other BCs, such transforms are available. We are talking of Periodic BCs, for which FFT is available to this purpose [2]. These BCs assume that the signal repeats itself periodically in both the two directions. So, considering for simplicity the $1 \mathrm{D}$ case, they impose that

$$
x_{1-j}=x_{n+1-j} \quad \text { and } \quad x_{n+j}=x_{j}, \quad j=1, \ldots, p,
$$

where $p$ is the parameter related to number of pixels outside the FOV that are taken into account. For multidimensional problems it is enough to apply the same assumption in every direction. The $2 \mathrm{D}$ corresponding blurring matrix $A$ is a BCCB matrix. We will use the symbol $C$ to denote this class of matrices. Clearly, it happens quite rarely that the image is periodic outside the FOV, so we often have ringing effects in restoration. The research of more accurate models has lead to define quite sophisticated BCs. A step in this direction is represented by Reflective BCs, which assume that the signal outside the FOV is a reflection of the signal inside the FOV. Formally, in the 1D case, these BCs impose that

$$
x_{1-j}=x_{j} \quad \text { and } \quad x_{n+j}=x_{n+1-j}, \quad j=1, \ldots, p .
$$

The corresponding blurring matrix $A$ is a Block Toeplitz plus Hankel with Toeplitz plus Hankel blocks, which can be diagonalized by Discrete Cosine Transform (DCT) when the PSF is symmetric [10]. We will use the symbol $\mathcal{R}$ to denote this class of matrices. Anti-Reflective BCs assume that the signal outside the FOV is an antireflection of the signal inside the FOV. Formally, in the 1D case, these BCs impose that

$$
x_{1-j}=2 x_{1}-x_{j+1} \quad \text { and } \quad x_{n+j}=2 x_{n}-x_{n-j}, \quad j=1, \ldots, p .
$$

The corresponding blurring matrix $A$ is a Block Toeplitz plus Hankel with Toeplitz plus Hankel blocks plus a low rank correction, which can be diagonalized by Anti-Reflective Transform (ART) when the PSF is symmetric [15]. We will use the symbol $\mathcal{A R}$ to denote this class of matrices. Both for Reflective and Anti-Reflective BCs a fast transform is available, but only if the PSF is quadrantally symmetric, i.e. symmetric in both horizontal and vertical direction. In the 2D case the same assumption on the image outside the FOV is done firstly in one direction and then in the other direction. For a detailed discussion on BCs and the associated blurring matrices please refer to $[2,16]$.

In summary, about the presented BCs, we have $A=\mathcal{T}_{n}(f)$ for Zero BCs, $A=\mathcal{C}_{n}(f)=\mathcal{T}_{n}(f)+\mathcal{B}_{n}^{C}(f)$ for Periodic BCs, $A=\mathcal{R}_{n}(f)=\mathcal{T}_{n}(f)+\mathcal{B}_{n}^{\mathcal{R}}(f)$ for Reflective BCs and $A=\mathcal{A R} \mathcal{R}_{n}(f)=\mathcal{T}_{n}(f)+\mathcal{B}_{n}^{\mathcal{P R}}(f)$ for Anti-Reflective BCs. We notice that in all these four cases $A$ has a Toeplitz structure $\mathcal{T}_{n}(f)$, given by the shift-invariant structure of the continuous operator, plus a correction $\mathcal{B}_{n}^{X}(f), \mathcal{X}=\mathcal{C}, \mathcal{R}, \mathcal{A R}$ depending on the BCs. Even if, as said, in some cases the BCs suffer from the lack of fast trigonometric transforms, for all of them the matrix-vector multiplication can be always computed in an efficient way by exploiting the structure $A=\mathcal{T}_{n}(f)+\mathcal{B}_{n}(f)$. Indeed, the multiplication can be made by means of FFTs (accessing only the PSF) on an appropriately padded image of larger size. In conclusion, we employ the unified notation $A=\mathcal{M}_{n}(f)$, where $\mathcal{M}$ can be any of the classes of matrices just introduced (i.e. $\mathcal{T}, C, \mathcal{R}, \mathcal{A R}$ ). This notation highlights the two crucial ingredients that form $A$ : the blurring phenomena associated to the PSF described by $f$ and the involved BCs represented by $\mathcal{M}$.

\section{Structure preserving reblurring preconditioning}

As said in the Introduction, usually iterative regularization methods show a low convergence rate. A way to remedy this drawback is to apply a (regularized) preconditioner $D$ and then to solve the linear system (3) equivalent to (2). With the aim to stabilize iterative methods and at the same time to speed up the convergence rate, in [5] we introduced a single preconditioning matrix $Z$ and replaced the preconditioned normal equations with the reblurring equation (4). This leads to reformulate iterative methods as the Landweber method in the new preconditioning context, that is to replace the following preconditioned iterative scheme

$$
x_{k+1}=x_{k}+\tau D A^{H}\left(b-A x_{k}\right)
$$


with

$$
x_{k+1}=x_{k}+\tau Z\left(b-A x_{k}\right),
$$

where $\tau$ is a positive relaxation parameter. In the following we fix $\tau=1$, by applying an implicit rescaling of the preconditioned system matrix $Z A$. Although $Z A$ is not in general symmetric, the convergence of the modified Landweber method (12) can be easily assured [5].

A way to build $Z$ is to apply a coarsening technique to the PSF of the problem (see [5]). Another more common way is to use filtering strategies. More in detail, in the case of periodic BCs, $Z$ is built as the BCCB matrix whose eigenvalues $v_{j}$ are obtained from the eigenvalues $\lambda_{j}$ of $A$ using some filtered inversion [17]. In particular, two very popular filters are the Hanke-Nagy-Plemmons (HNP) filter [18], defined as

$$
v_{j}=\left\{\begin{array}{cl}
\bar{\lambda}_{j} /\left|\lambda_{j}\right|^{2}, & \text { if }\left|\lambda_{j}\right| \geq \zeta, \\
\bar{\lambda}_{j}, & \text { if }\left|\lambda_{j}\right|<\zeta,
\end{array} \quad j=1, \ldots, n^{2},\right.
$$

and the Tikhonov filter, defined as

$$
v_{j}=\frac{\bar{\lambda}_{j}}{\left|\lambda_{j}\right|^{2}+\alpha}, \quad j=1, \ldots, n^{2},
$$

where $\alpha$ and $\zeta$ are positive regularization parameters. Clearly, in case of periodic BCs, since we are in the circulant algebra, the classical preconditioning approach (based on $D$ ) and this new one (based on $Z$ ) are the same thing. However, for other BCs, the $Z$ variant shows better performance and higher stability (in relation to the choice of regularization parameters, e.g. $\alpha$ for Tikhonov) than standard preconditioning (see [5]). Thus, for any BCs, $Z$ is built as the BCCB matrix obtained by applying filtering to the same PSF (i.e. the same generating function $f$ ) that gives rise to the matrix $A$. In other words, if we call $g$ such filtered function, recalling the notation introduced in Section 2, we have that $A=\mathcal{M}_{n}(f)$ and $Z=C_{n}(g)$.

Regardless of the BCs adopted to build the blurring matrix $A$, in [5] $Z$ is always a BCCB matrix. The main new idea of this paper is to build $Z$ so that it inherits the same matrix structure of $A$. To do that we compute the eigenvalues $c_{i, j}$ of $C_{n}(f)$, the $n^{2} \times n^{2}$ BCCB matrix associated with the PSF $H$, by means of the two-dimensional FFT of $H$. By definition

$$
c_{i, j}=f\left(\frac{2 \pi i}{n}, \frac{2 \pi j}{n}\right), \quad i, j=0, \ldots, n-1,
$$

where $f$ is the generating function (6) of the blurring matrix $A=A(f)$.

We can now regularize such eigenvalues. Let us denote by $v_{i, j}$ the values obtained by applying the Tikhonov filter (14) to $c_{i, j}$, instead of $\lambda_{j}$. Since it usually gives very good (and often the best) numerical results, we simply consider the Tikhonov filter (14). However, any other filter could be applied as well. On the ground of the theory of eigenvalues decomposition of BCCB matrices, the values $v_{i, j}$ can be considered as a sampling of the function

$$
g\left(x_{1}, x_{2}\right)=\sum_{j_{1}, j_{2}=-\left\lfloor\frac{n+1}{2}\right\rfloor}^{\left\lfloor\frac{n-1}{2}\right\rfloor} \beta_{j_{1}, j_{2}} \mathrm{e}^{\hat{\imath}\left(j_{1} x_{1}+j_{2} x_{2}\right)}
$$

at the grid points $\Omega_{n}=\left\{\left(\frac{2 \pi i}{n}, \frac{2 \pi j}{n}\right) \mid i, j=0, \ldots, n-1\right\}$ (see [14]). Namely,

$$
g\left(\frac{2 \pi i}{n}, \frac{2 \pi j}{n}\right):=v_{i, j}=\frac{\bar{c}_{i, j}}{\left|c_{i, j}\right|^{2}+\alpha}=\frac{\bar{f}\left(\frac{2 \pi i}{n}, \frac{2 \pi j}{n}\right)}{\left|f\left(\frac{2 \pi i}{n}, \frac{2 \pi j}{n}\right)\right|^{2}+\alpha} .
$$

Note that $g$ is a regularized approximation of the inverse of $f$ on $\Omega_{n}$. The function $g$ is univocally identified by the $n^{2}$ interpolation conditions (17), for $i, j=0, \ldots, n-1$, and its coefficients $\beta_{j_{1}, j_{2}}$ can be computed by means of a two-dimensional IFFT of these values $g\left(\frac{2 \pi i}{n}, \frac{2 \pi j}{n}\right)$. We will denote by $\widetilde{H}$ the mask of the coefficients $\beta_{j_{1}, j_{2}}, j_{1}, j_{2}=-\left\lfloor\frac{n+1}{2}\right\rfloor, \ldots,\left\lfloor\frac{n+1}{2}\right\rfloor$. It is worth observing that up to this point the described technique is just like the one proposed in [5]. The main difference between this approach and that described in [5] is that in the latter the function $g$ is used as the symbol of a BCCB matrix, while here we combine $g$ with the BCs of the problem defining a matrix

$$
Z:=\mathcal{M}_{n}(g)
$$


that has the same structure of the original system matrix $A=M_{n}(f)$ of our blurring model (1).

The following algorithm summarizes how to build our structure preserving preconditioner.

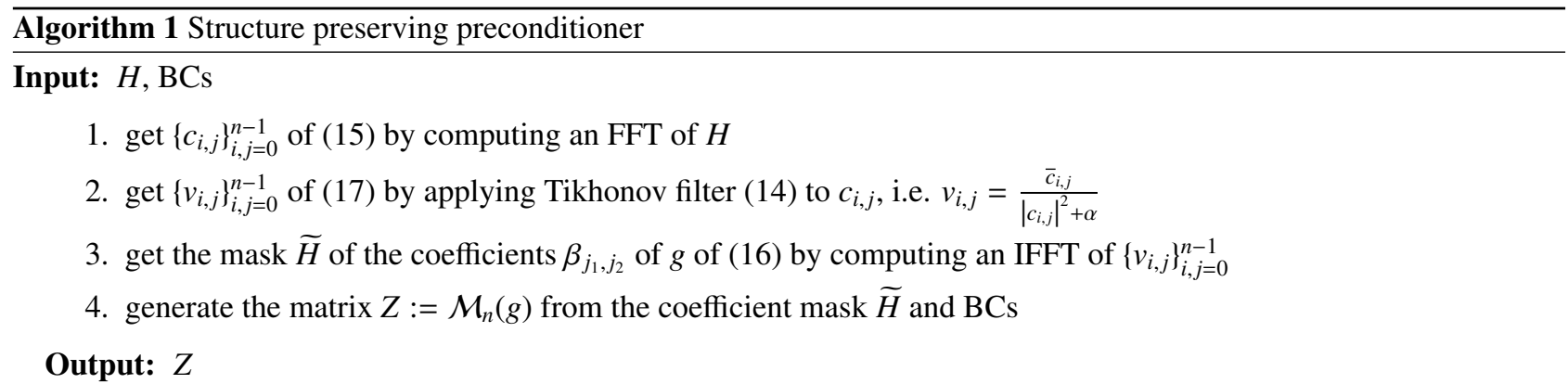

Throughout, we refer to the circulant preconditioning technique proposed in [5] as $Z_{\text {circ }}$, while our structure preserving preconditioner will be denoted by $Z_{\text {struct }}$. Note that when the PSF is quadrantally symmetric, i.e. $h_{i, j}=h_{ \pm i, \pm j}$, then the generating function $f$ is a cosine polynomial thanks to the Eulero formula. Therefore, it is worthwhile looking for $g$ in (17) as a cosine polynomial, which implies that Algorithm 1 can be reformulated by replacing FFT with DCT.

Furthermore it is interesting to note that, by appropriately changing the filter at step 2, Algorithm 1 can be used for creating a structured preconditioner $D$ (instead of $Z$ ). For instance, considering the linear system (3), $D$ has the role of preconditioning $A^{H} A$, so a filter that can be used for creating $D$ is the following one: $v_{i, j}=\frac{1}{\left|c_{i, j}\right|^{2}+\alpha}$. However, such preconditioning strategy applied to Landweber method is inferior to the $Z$ variant of such method.

If we take into account the Conjugate Gradient for Least Squares (CGLS), for this method it is available a preconditioned version (PCGLS) associated with the following linear system

$$
D^{\frac{1}{2}} A^{H} A D^{\frac{1}{2}} y=D^{\frac{1}{2}} A^{H} b, \quad y=D^{-\frac{1}{2}} x,
$$

where $D$ is a positive definite matrix that has the role of preconditioning $A^{H} A$. Therefore a filter that can be used for creating $D^{\frac{1}{2}}$ is the next one: $v_{i, j}=\frac{1}{\sqrt{\left|c_{i, j}\right|^{2}+\alpha}}$. It is known that classical circulant preconditioning applied to CGLS is able to speed up the method, but usually it negatively affects the restoration accuracy. As it can be seen by numerical evidences reported in this work, by using structured preconditioners it is possible to overcome this issue.

\subsection{Comparison with Hanke-Nagy preconditioner}

For Zero BCs and symmetric PSF we can seek a strict link between the proposed structure preserving preconditioner $Z_{\text {struct }}$ and the Toeplitz preconditioning proposed by Hanke and Nagy in [9] for real symmetric Toeplitz systems. For simplicity, in analyzing the analogies and the differences between these techniques, we consider the one-dimensional case. Our aim in this subsection is to study, in a sense that will be further explained, how much the two preconditioners are close. To recognize that the PSF $H$ is symmetric, we fix the central pixel at the center of $H$. Moreover, we assume $n$ even for the sake of simplicity in the computations. Therefore, the 1D PSF is $H=\left[h_{\frac{n}{2}-1}, \ldots, h_{0}, \ldots, h_{\frac{n}{2}-1}, 0\right]$ and the associated generating function is

$$
f(x)=h_{0}+2 \sum_{j=1}^{\frac{n}{2}-1} h_{j} \cos (j x),
$$

obtaining the $n \times n$ blurring matrix $A=\mathcal{T}_{n}(f)$ in the Toeplitz case, which is related to Zero BCs.

We briefly recall the computation of the preconditioner proposed in [9]. First of all, the matrix $\mathcal{T}_{n}(f)$ is embedded in the symmetric circulant $2 n \times 2 n$ matrix

$$
C_{2 n}(f)=\left(\begin{array}{cc}
\mathcal{T}_{n}(f) & R \\
R & \mathcal{T}_{n}(f)
\end{array}\right)
$$

whose first column is given by $\left[h_{0}, \ldots, h_{\frac{n}{2}-1}, 0, \ldots, 0, h_{\frac{n}{2}-1}, \ldots, h_{1}\right]^{T}$. Then, the eigenvalues of $C_{2 n}$, computed via FFT, are inverted by means of the HNP regularization filter (13) in order to obtain a regularized inverse of $C_{2 n}$. Finally the preconditioner is selected as the first $n \times n$ principal submatrix of such a $2 n \times 2 n$ regularized inverse of $C_{2 n}$.

The following proposition summarizes our result. 
Proposition 1. Assume a family $\left(\mathcal{T}_{n}(f)\right)_{n}$ of $n \times n$ image deblurring matrices with Zero BCs, where $f$ denotes the generating function (19) of a real fully-symmetric PSF. For any matrix $\mathcal{T}_{n}(f)$, let $Z_{\text {struct }, n}$ denote the associated structure preserving preconditioner of Algorithm 1 and $P_{\mathrm{HN}, n}$ denote the associated inverse Toeplitz preconditioner by Hanke and Nagy [9], both regularized by the same Tikhonov filter (14). Then the two preconditioners $Z_{\mathrm{struct}, n}$ and $P_{\mathrm{HN}, n}$ are asymptotically equivalent, i.e. holds the following result

$$
\left\|Z_{\mathrm{struct}, n}-P_{\mathrm{HN}, n}\right\| \underset{n \rightarrow \infty}{\longrightarrow} 0,
$$

where $\|\bullet\|$ denotes the spectral norm of matrices. In particular, $\left\|Z_{\mathrm{struct}, n}-P_{\mathrm{HN}, n}\right\|=\mathrm{O}\left(\log (n) \mathrm{e}^{-c n}\right)$, with $c>0$.

Proof. As first step we explicitly compute the $Z_{\text {struct }, n}$ preconditioner of $\mathcal{T}_{n}(f)$. Algorithm 1 considers the symmetric circulant matrix $C_{n}(f)$ whose first column is $\left[h_{0}, \ldots, h_{\frac{n}{2}-1}, 0, h_{\frac{n}{2}-1}, \ldots, h_{1}\right]^{T}$. Hence, the step 1 of the algorithm computes the eigenvalues of $C_{n}(f)$ that are

$$
c_{k}=f\left(\frac{2 k \pi}{n}\right)=h_{0}+2 \sum_{j=1}^{\frac{n}{2}-1} h_{j} \cos \left(\frac{2 j k \pi}{n}\right), \quad k=0, \ldots, n-1 .
$$

At step 2 we adopt the Tikhonov filter (14), so that we compute the regularized inverses $v_{k}$ of the eigenvalues as

$$
v_{k}=\frac{\bar{c}_{k}}{c_{k}^{2}+\alpha}, \quad k=0, \ldots, n-1 .
$$

We can write $v_{k}=g\left(\frac{2 k \pi}{n}\right), k=0, \ldots, n-1$, where $g \in \widetilde{\Pi}_{n-1}=\operatorname{span}\{\cos (j x), j=0, \ldots, n-1\}$ is the trigonometric interpolating polynomial on the pairs $\left(\frac{2 k \pi}{n}, v_{k}\right), k=0, \ldots, n-1$, that is

$$
g(x)=\beta_{0}+2 \sum_{j=1}^{n-1} \beta_{j} \cos (j x)
$$

Actually, it can be easily proved that $g$, although it depends on $n$ interpolation conditions, has degree $n / 2$. Indeed, by rewriting the interpolation conditions as

$$
\begin{aligned}
\left(\frac{2 k \pi}{n}, v_{k}\right) & k & =0, \ldots, \frac{n}{2} \\
\left(\frac{(n / 2+k) \pi}{n / 2}, v_{n / 2+k}\right) & k & =1, \ldots, \frac{n}{2}-1
\end{aligned}
$$

and observing that $v_{k}=v_{n-k}$, for $k=1, \ldots, \frac{n}{2}-1$, from (21) and the symmetry of $g$ in (22) with respect to the interval $[0,2 \pi]$, we have that $g \in \widetilde{\Pi}_{\frac{n}{2}}$. For this reason in the following we rename $g$ as $g_{\frac{n}{2}}$. The steps 3 and 4 build the preconditioner as the matrix that preserves the structure of $A$ and whose symbol is $g_{\frac{n}{2}}$, hence for Zero BCs, the preconditioner finally is

$$
Z_{\text {struct }, n}=\mathcal{T}_{n}\left(g_{\frac{n}{2}}\right) .
$$

Now we consider the $P_{\mathrm{HN}, n}$ preconditioner of $\mathcal{T}_{n}(f)$. The eigenvalues of the $2 n \times 2 n$ circulant matrix $C_{2 n}(f)$ defined by (20) are

$$
\mu_{k}=f\left(\frac{2 k \pi}{2 n}\right)=f\left(\frac{k \pi}{n}\right), \quad k=0, \ldots, 2 n-1 .
$$

Applying again the Tikhonov filter (14) (unlike the HNP filter considered in [9]), we obtain the regularized inverses $u_{k}$ of the eigenvalues as follows

$$
u_{k}=\frac{\bar{\mu}_{k}}{\mu_{k}^{2}+\alpha}, \quad k=0, \ldots, 2 n-1 .
$$

By means of an analogous reasoning to that performed before, it can be shown that there exists a unique polynomial $h_{n} \in \widetilde{\Pi}_{n}$ that interpolates the $2 n$ pairs $\left(\frac{k \pi}{n}, u_{k}\right), k=0, \ldots, 2 n-1$. In fact, due to their symmetry, among the previous interpolation conditions only the one relating to $k=0, \ldots, n$ are distinct. The preconditioner $P_{\mathrm{HN}, n}$ is then selected as the first $n \times n$ principal submatrix of the $2 n \times 2 n$ circulant matrix $C_{2 n}\left(h_{n}\right)$. Denoted by $S$ the $2 n \times n$ matrix whose $j^{\text {th }}$ column is the $j^{\text {th }}$ element of the canonical basis of $\mathbb{R}^{2 n}$, the preconditioner can be expressed as

$$
P_{\mathrm{HN}, n}=\mathcal{T}_{n}\left(h_{n}\right)=S^{H} C_{2 n}\left(h_{n}\right) S .
$$


We can now relate the two preconditioners $Z_{\mathrm{struct}, n}=\mathcal{T}_{n}\left(g_{\frac{n}{2}}\right)$ and $P_{\mathrm{HN}, n}=\mathcal{T}_{n}\left(h_{n}\right)$. Let us observe that defining

$$
\psi(x)=\frac{\bar{f}(x)}{f^{2}(x)+\alpha},
$$

we have that $g_{\frac{n}{2}}$ interpolates $\psi$ on $\Omega_{\frac{n}{2}}=\left\{\frac{2 k \pi}{n}, \quad k=0, \ldots, \frac{n}{2}\right\}$ and $h_{n}$ interpolates the same $\psi$ on $\Omega_{n}=\left\{\frac{k \pi}{n}, \quad k=0, \ldots, n\right\}$. Since $\Omega_{\frac{n}{2}} \subset \Omega_{n}$ and $h_{n} \in \widetilde{\Pi}_{n}$, then we can write

$$
h_{n}=g_{\frac{n}{2}}+p_{n}
$$

where $p_{n} \in \widetilde{\Pi}_{n}$ such that $p_{n}\left(\frac{2 k \pi}{n}\right)=0$, for $k=0, \ldots, \frac{n}{2}$. On this ground we rewrite

$$
p_{n}=E_{\frac{n}{2}}-E_{n}
$$

where $E_{\frac{n}{2}}=\psi-g_{\frac{n}{2}}$ and $E_{n}=\psi-h_{n}$ are the classical remainder in the interpolation of $\psi$ on $\Omega_{\frac{n}{2}}$ and $\Omega_{n}$, respectively. By virtue of the linearity of $\mathcal{T}_{n}$, we have

$$
\begin{aligned}
\left\|\mathcal{T}_{n}\left(g_{\frac{n}{2}}\right)-\mathcal{T}_{n}\left(h_{n}\right)\right\| & =\left\|\mathcal{T}_{n}\left(g_{\frac{n}{2}}\right)-\mathcal{T}_{n}\left(g_{\frac{n}{2}}\right)-\mathcal{T}_{n}\left(p_{n}\right)\right\|=\left\|\mathcal{T}_{n}\left(E_{\frac{n}{2}}-E_{n}\right)\right\| \\
& \leq\left\|E_{\frac{n}{2}}\right\|_{\infty}+\left\|E_{n}\right\|_{\infty},
\end{aligned}
$$

from well-known properties of Toeplitz matrices, cf. [19], where $\|\cdot\|_{\infty}$ denotes the spectral norm of matrices and $\|\cdot\|_{\infty}$ is the classical supremum norm of functions in $[0,2 \pi]$. By construction, $E_{n}$ is the remainder function of the interpolation of $\psi$ on the $n+1$ Chebyshev-Lobatto nodes defined as $\cos \left(\frac{k \pi}{n}\right)$ for $k=0, \ldots, n$. Its Lebesgue constant is known to grow as $k_{1} \log (n)$ where $k_{1}$ is a constant. Thus, $\left\|E_{n}\right\|_{\infty}$ can be bounded in the following way

$$
\left\|E_{n}\right\|_{\infty} \leq k_{1} \log (n)\left\|a_{n}\right\|_{\infty}
$$

where $\left\|a_{n}\right\|_{\infty}$ is the error in the best approximation of $\psi$ in the space of polynomials of degree at most $n$. Applying a similar reasoning to $E_{\frac{n}{2}}$, we can bound it as $\left\|E_{\frac{n}{2}}\right\|_{\infty} \leq k_{2} \log \left(\frac{n}{2}\right)\left\|a_{\frac{n}{2}}\right\|_{\infty}$. Because of the $C^{\infty}$ regularity of $\psi$, $\left\|a_{r}\right\|$ is exponentially converging to zero as $r$ tends to $+\infty$ by Bernstein Theorem. In conclusion, from (27) it follows that $\| \mathcal{T}_{n}\left(g_{\frac{n}{2}}\right)-$ $\mathcal{T}_{n}\left(g_{n}\right) \|=\mathrm{O}\left(\log (n) \mathrm{e}^{-c n}\right)$, with $c>0$, i.e. the two preconditioners $Z_{\text {struct }, n}=\mathcal{T}_{n}\left(g_{\frac{n}{2}}\right)$ and $P_{\mathrm{HN}, n}=\mathcal{T}_{n}\left(h_{n}\right)$ are asymptotically equivalent.

It is interesting to notice that the equivalence result of Proposition 1 is confirmed by several numerical tests, where basically we got the same deblurring accuracy and the same convergence speed by applying the two preconditioners. On the other hand, from a computational point of view $Z_{\text {struct }, n}$ of (23) requires two FFTs of size $n$ instead of two FFTs of size $2 n$ to obtain $P_{\mathrm{HN}, n}$ of (24).

\section{Non-stationary preconditioning}

When we deal with a stationary regularization method we have always to face the non-trivial task of determining a good choice for the filtering parameter $\alpha$. In [12] the authors proposed a non-stationary version of the $Z$ reblurring preconditioner where the parameter $\alpha$ is dynamically estimated at every iteration instead of to be fixed a-priori. The iteration is the following

$$
x_{k+1}=x_{k}+Z_{\mathrm{circ}}^{k} r_{k}, \quad Z_{\mathrm{circ}}^{k}=C^{*}\left(C C^{*}+\alpha_{k} I\right)^{-1}, \quad r_{k}=b-A x_{k},
$$

where $C=C_{n}(f)$ is the BCCB matrix associated with $H$. Note that if $\alpha_{k}=\alpha$ then the iteration (28) is exactly (12) with $Z=C_{n}(g)$, where $g$ is defined in (16) imposing (17) as in [5]. $Z_{\text {circ }}^{k}$ is simply the BCCB matrix obtained by Algorithm 1 with $\alpha_{k}$ in place of $\alpha$ and with periodic BCs.

In [12], the iteration-dependent regularization parameter $\alpha_{k}$ is obtained by solving the following non-linear equation

$$
\left\|r_{k}-C Z_{\mathrm{circ}}^{k} r_{k}\right\|=q_{k}\left\|r_{k}\right\|, \quad 0<q_{k}<1,
$$

with a few steps of the Newton iteration. Here the parameter $q_{k}$ depends on the noise level and it is related to a value $0<\rho_{\text {circ }}<1 / 2$ which satisfies the assumption

$$
\|(C-A) z\| \leq \rho_{\text {circ }}\|A z\|, \quad \forall z \in \mathbb{R}^{n} .
$$


This parameter $\rho_{\text {circ }}$ measures how much we trust in the approximation of $A$ with its BCCB counterpart $C$; the smaller $\rho_{\text {circ }}$ is, the more we trust in that approximation (see [12] for more details). From a numerical point of view, the parameter $\rho_{\text {circ }}$ is usually chosen among the values $\left\{10^{-1}, 10^{-2}, 10^{-3}, 10^{-4}\right\}$. A too small $\rho_{\text {circ }}$ can be easily recognized looking at the sequence of the residual norms. The iterations are stopped under a special choice of the discrepancy principle that will be discussed later. From a theoretical point of view, in [12] it is proved that under the assumption (29), the iteration (28) converge monotonically and it is a regularization method.

In this section we extend the non-stationary iteration (28) to take into account the BCs of the problem following our structure preserving strategy. More precisely, we consider the following iteration

$$
x_{k+1}=x_{k}+Z_{\text {struct }}^{k} r_{k}, \quad r_{k}=b-A x_{k},
$$

where $Z_{\text {struct }}^{k}$ is the structure preserving matrix built by means of Algorithm 1 with a special choice of the regularization parameter $\alpha_{k}$. In practice, we would estimate $\alpha_{k}$ by solving

$$
\left\|r_{k}-A Z_{\text {struct }}^{k} r_{k}\right\|=q_{k}\left\|r_{k}\right\|
$$

which is not computationally practicable. Therefore, we estimate the regularization parameter $\alpha_{k}$ using an approximation of (32) easily computable that is the equation (29). A similar strategy is used in [13] for different regularization methods. Note that the two iterations (28) and (31) retrieve a different sequence of $\alpha_{k}$, even if both use the equation (29) to estimate $\alpha_{k}$, because the sequences of the residuals $\left\{r_{k}\right\}_{k}$ in the two iterative schemes are different. Furthermore, we have to observe that for such a modified version of (28) the condition (30) does not make sense and then we are allowed to introduce a new parameter $\rho_{\text {struct }}$ whose value could not match with the one of $\rho_{\text {circ }}$. Note that since $Z_{\text {struct }}^{k}$ provides a better approximation of $A^{*}\left(A A^{*}+\alpha_{k} I\right)^{-1}$ with respect to $Z_{\text {circ }}^{k}$, it is expected that $\rho_{\text {struct }}<\rho_{\text {circ }}$. On the other hand, we cannot prove convergence results as in [12], even if the numerical results in Section 5 shows that our structured non-stationary preconditioning is robust and very effective.

As shown in [12], another suitable choice of the parameter $\alpha_{k}$ for iteration (28) is given by the geometric sequence

$$
\alpha_{k}=\widetilde{\alpha} q^{k}, \quad k=0,1, \ldots,
$$

where $\widetilde{\alpha}>0$ and $0<q<1$. In the next section we confirm the effectiveness of the iteration (31) with both sequences of $\left\{\alpha_{k}\right\}$ obtained by (29) or (33).

\section{Numerical results}

In this section we provide some numerical tests for the image deblurring problem of type (1). In each example we impose appropriate BCs and solve the linear system $A x=b$ using both stationary and non-stationary preconditioned iterations (12) and (31). More in detail, in the stationary case, fixed few values of the parameter $\alpha$, we compare the performance of the preconditioner $Z_{\text {circ }}$ with $Z_{\text {struct }}$. In the non-stationary case our attention is devoted to the comparison between preconditioners $Z_{\text {circ }}^{k}$ and $Z_{\text {struct }}^{k}$. Regarding the sequence $\left\{\alpha_{k}\right\}_{k}$ of the regularization parameter, we investigate the behaviour of both the geometric sequence defined in (33) (labeled 'geometric' in the following) and the sequence computed solving (29) (throughout, labeled 'DH' (Donatelli-Hanke)). For the geometric sequence we fix $\widetilde{\alpha}=0.5$ and $q=0.7$ in (33), as suggested in [12]. Furthermore, in our tests we consider also CGLS and PCGLS, the latter employing a structured preconditioner created by means of Algorithm 1 with a proper filter, as described in Section 3 . The choice of $\alpha$ related to this filter, which in general is a problematic issue, is made manually.

We use the well-known discrepancy principle as stopping criterion for the iterative methods, i.e. we stop them at the first iteration $m$ that satisfies the condition

$$
\left\|A x_{m}-b\right\|_{2}<\gamma\|\eta\|_{2}
$$

where $x_{m}$ is the approximation provided by the method at the $m$-th iteration and $\gamma \geq 1$. In the following we fix $\gamma=$ $\left(1+2 \rho_{\text {circ }}\right) /\left(1-2 \rho_{\text {circ }}\right)$ or $\gamma=\left(1+2 \rho_{\text {struct }}\right) /\left(1-2 \rho_{\text {struct }}\right)$ accordingly to the choice of the preconditioner $\left(Z_{\text {circ }}^{k}\right.$ or $\left.Z_{\text {struct }}^{k}\right)$ for the iterations (28) and (31) with the DH sequence of regularization parameters (see [12]), while we choose $\gamma=1.01$ for the other algorithms. The initial guess $x_{0}$ is always taken as the observed image $b$. Assuming to know the true image solution $\bar{x}$ of (1), we measure the quality of the $k$-th iteration $x_{k}$ by computing the Relative Restoration Error (RRE) defined as $\mathrm{RRE}=\left\|\bar{x}-x_{k}\right\|_{2} /\|\bar{x}\|_{2}$. We refer to the minimum RRE and to the RRE corresponding to the discrepancy principle iteration as $R_{R E}$ min and $R_{R E}$ discr, respectively. All the numerical tests have been developed with MATLAB. 


\subsection{Example 1}

We start with the deblurring problem reported in Figure 1, The PSF is a motion blur and the noise level is $1 \%$. In this example we impose Reflective BCs. In Table 1 we compare $Z_{\text {struct }}, Z_{\text {circ }}$ and $Z_{\mathrm{DCT}}$ preconditioners for $\alpha=$ $0.5,0.1,0.05,0.01$. We remind that $Z_{\mathrm{DCT}}$ is a structured preconditioner as $Z_{\text {struct }}$, but unlike $Z_{\text {struct }}$ it is built from a symmetrized version of the original PSF, which allows to get the eigenvalues by DCT. As expected, since the PSF is highly non-symmetric, $Z_{\mathrm{DCT}}$ shows very poor performance, so we do not consider it further in this example. We observe that both $\mathrm{RRE}_{\min }$ and $\mathrm{RRE}_{\text {discr }}$ provided by $Z_{\text {struct }}$ preconditioner are smaller than the $\mathrm{RRE}_{\min }$ obtained using the $Z_{\text {circ }}$ one. Furthermore, the discrepancy principle does not work for $Z_{\text {circ }}$ preconditioner when $\alpha=0.5,0.1,0.05$ (shown as - symbol in Table 1).

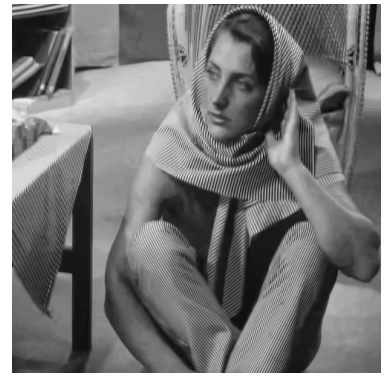

(a)

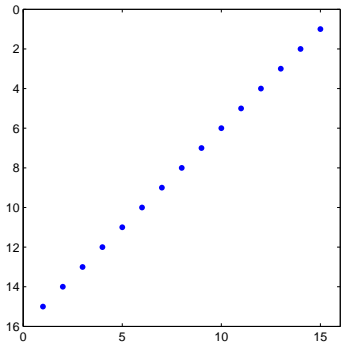

(b)

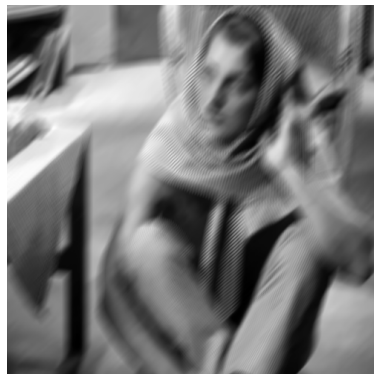

(c)

Figure 1: Example 1: (a) true image; (b) PSF; (c) blurred and noisy image.

\begin{tabular}{|c|c|c||c|c||c|c||c|c|}
\cline { 2 - 9 } \multicolumn{1}{c|}{} & \multicolumn{2}{c||}{$\alpha=0.5$} & \multicolumn{2}{c||}{$\alpha=0.1$} & \multicolumn{3}{c|}{$\alpha=0.05$} & \multicolumn{3}{c|}{$\alpha=0.01$} \\
\cline { 2 - 9 } \multicolumn{1}{c|}{} & $\mathrm{RRE}_{\min }$ & $\mathrm{RRE}_{\text {discr }}$ & $\mathrm{RRE}_{\min }$ & $\mathrm{RRE}_{\text {discr }}$ & $\mathrm{RRE}_{\min }$ & $\mathrm{RRE}_{\text {discr }}$ & $\mathrm{RRE}_{\min }$ & $\mathrm{RRE}_{\text {discr }}$ \\
\hline$Z_{\text {struct }}$ & $0.1084(46)$ & $0.1093(34)$ & $0.1072(10)$ & $0.1077(8)$ & $0.1068(5)$ & $0.1068(5)$ & $0.1070(1)$ & $0.1084(2)$ \\
\hline$Z_{\text {circ }}$ & $0.1138(31)$ & $-(-)$ & $0.1125(7)$ & $-(-)$ & $0.1115(4)$ & $-(-)$ & $0.1096(1)$ & $0.1145(2)$ \\
\hline$Z_{\text {DCT }}$ & $0.1446(4)$ & $-(-)$ & $0.1422(2)$ & $-(-)$ & $0.1396(1)$ & $-(-)$ & $0.1361(1)$ & $-(-)$ \\
\hline
\end{tabular}

Table 1: Example 1: $\mathrm{RRE}_{\min }$ and $\mathrm{RRE}_{\mathrm{discr}}$ and corresponding iterations (in parentheses) for $Z_{\text {struct }}, Z_{\text {circ }}$ and $Z_{\mathrm{DCT}}$ preconditioners.

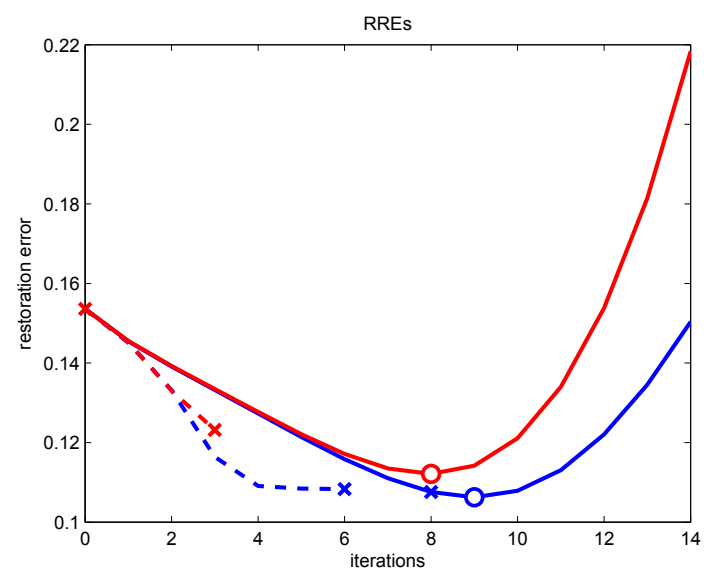

(a) $\rho_{\text {circ }}=10^{-1}, \rho_{\text {struct }}=10^{-2}$

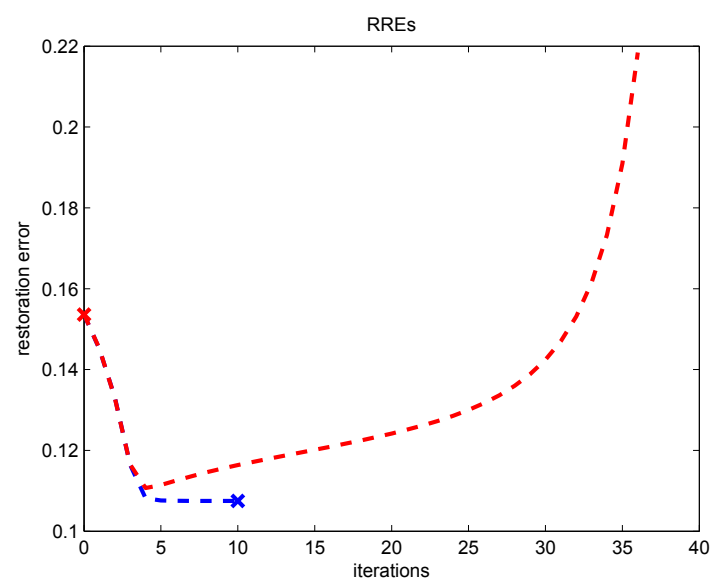

(b) $\rho_{\text {circ }}=10^{-2}, \rho_{\text {struct }}=10^{-3}$

Figure 2: Example 1: (a) Comparisons between RREs for $Z_{\text {struct }}^{k}$ geometric (solid blue line), $Z_{\text {circ }}^{k}$ geometric (solid red line), $Z_{\text {struct }}^{k}$ DH (dashed blue line) and $Z_{\text {circ }}^{k} \mathrm{DH}$ (dashed red line) for $\rho_{\text {struct }}=10^{-2}$ and $\rho_{\text {circ }}=10^{-1}$; (b) Comparison between $Z_{\text {struct }}^{k}$ DH (dashed blue line) and $Z_{\text {circ }}^{k}$ DH (dashed red line) for $\rho_{\text {struct }}=10^{-3}$ and $\rho_{\text {circ }}=10^{-2}$. Key to symbols: $(\circ)$ optimal iteration, $(\times)$ discrepancy iteration.

Figures 2(a)(b) refer to the comparison of the non-stationary preconditioning with $Z_{\text {struct }}^{k}$ for both geometric and DH sequences with the $Z_{\text {circ }}^{k}$ preconditioner. When possible, together with the discrepancy iteration, we also show the iteration corresponding to the $\mathrm{RRE}_{\text {min }}$ (by construction for $Z_{\text {circ }}^{k}$ and $Z_{\text {struct }}^{k}$ with $\mathrm{DH}$ sequence only $\mathrm{RRE}_{\text {discr }}$ is available). In the 
non-stationary $Z_{\text {circ }}^{k}$ context we fix $\rho_{\text {circ }}=10^{-1}$. Such a choice of $\rho_{\text {circ }}$ is due to the fact that for smaller values of this parameter, the $Z_{\text {circ }}^{k}$ method does not converge (see the behaviour of the RRE for $Z_{\text {circ }}^{k}$ with DH sequence in Figure 2(b) in which $\rho_{\text {circ }}=10^{-2}$ ). Regarding the parameter $\rho_{\text {struct }}$, we use both $10^{-2}$ and $10^{-3}$ and observe that the $Z_{\text {struct }}^{k}$ preconditioner works well for both choices of $\rho_{\text {struct }}$. Note that the previous behaviour of the parameter $\rho_{\text {circ }}$ and $\rho_{\text {struct }}$ agree with the remark made in Section 4. This highlights an appreciable stability of our algorithm with respect to the parameter $\rho_{\text {struct }}$. Although $Z_{\text {struct }}^{k}$ is slightly more accurate for $\rho_{\text {struct }}=10^{-3}\left(\mathrm{RRE}_{\mathrm{discr}}=0.1075\right)$ than for $\rho_{\text {struct }}=10^{-2}\left(\mathrm{RRE}_{\mathrm{discr}}=0.1083\right)$ (compare also Figure 2(a) with Figure 2(b)), we decide for the last one since in this case the non-stationary structure preserving method is faster (6 iterations rather than 10 iterations).

The better performance of $Z_{\text {struct }}^{k}$ with respect to $Z_{\text {circ }}^{k}$ can be appreciated again in Table 2 (within 'n/a' means that the corresponding $\mathrm{RRE}_{\min }$ is not available). In such table are also reported results relative to CGLS and PCGLS (with $\alpha=0.05$ ), which is able to get restorations of accuracy slightly better than CGLS in less steps. It is clear that not only the $\mathrm{RRE}_{\min }$, but also the $\mathrm{RRE}_{\text {discr }}$ of both $Z_{\text {struct }}^{k}$ algorithms (geometric and DH) are smaller than the RRE $\mathrm{E}_{\text {min }}$ provided by CGLS, PCGLS and $Z_{\text {circ }}^{k}$. We stress that the discrepancy principle does not work for the $Z_{\text {circ }}^{k}$ preconditioner with geometric sequence for $\alpha=0.5,0.1,0.05$. A comparison between the restorations in Figures 3(a)(c) and the ones in Figures 3(b)(d) highlights the effectiveness of the proposed non-stationary structure preserving preconditioners.

\begin{tabular}{|c|c|c||c|c||c|c|}
\cline { 2 - 7 } \multicolumn{1}{c|}{} & \multicolumn{2}{c|}{ geometric } & \multicolumn{2}{c||}{ DH } & \multirow{2}{*}{ CGLS } & \multirow{2}{*}{ PCGLS } \\
\cline { 2 - 7 } \multicolumn{1}{c|}{} & $Z_{\text {struct }}^{k}$ & $Z_{\text {circ }}^{k}$ & $Z_{\text {struct }}^{k}$ & $Z_{\text {circ }}^{k}$ & & \\
\hline$R^{2} E_{\min }$ & $0.1063(9)$ & $0.1121(8)$ & n/a & n/a & $0.1128(9)$ & $0.1115(2)$ \\
\hline RRE $_{\text {discr }}$ & $0.1076(8)$ & $-(-)$ & $0.1083(6)$ & $0.1232(3)$ & $0.1164(12)$ & $0.1161(3)$ \\
\hline
\end{tabular}

Table 2: Example 1: $\mathrm{RRE}_{\mathrm{min}}, \mathrm{RRE}_{\mathrm{discr}}$ and corresponding iterations (in parentheses) for $Z_{\text {struct }}^{k}, Z_{\text {circ }}^{k}$ preconditioners for both the geometric and DH sequences and for CGLS and PCGLS. We fix $\rho_{\text {struct }}=10^{-2}$ and $\rho_{\text {circ }}=10^{-1}$.

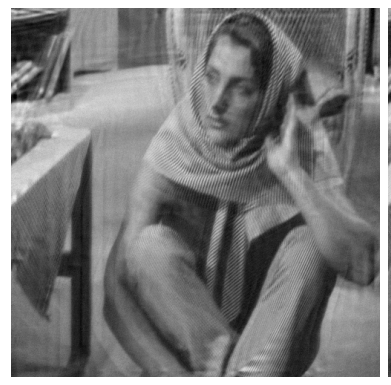

(a) $Z_{\text {struct }}^{k}$ geometric

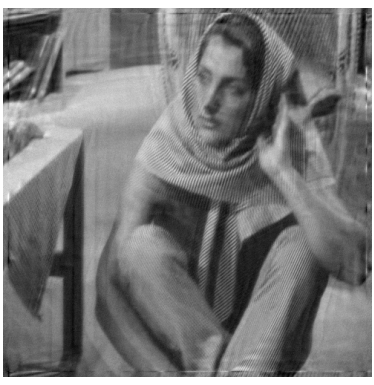

(b) $Z_{\text {circ }}^{k}$ geometric

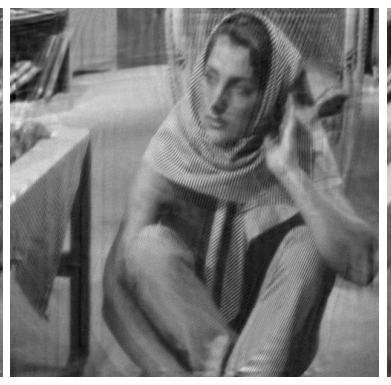

(c) $Z_{\text {struct }}^{k} \mathrm{DH}$

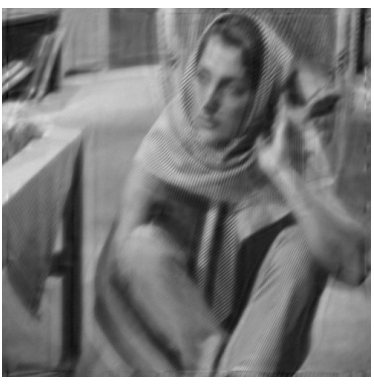

(d) $Z_{\text {circ }}^{k} \mathrm{DH}$

Figure 3: Example 1: (a) Discrepancy reconstruction with $Z_{\text {struct }}^{k}$ geometric; (b) Optimal reconstruction with $Z_{\text {circ }}^{k}$ geometric; (c) Discrepancy reconstruction with $Z_{\text {struct }}^{k} \mathrm{DH}$; (d) Discrepancy reconstruction with $Z_{\text {circ }}^{k} \mathrm{DH}$.

\subsection{Example 2}

The second example is the deblurring problem reported in Figure 4. The PSF is not far from being symmetric and the noise level is $0.2 \%$. We choose to employ Anti-Reflective BCs. In Table 3, we fix $\alpha=0.01,0.005,0.001,0.0005$ and show the $\mathrm{RRE}_{\min }, \mathrm{RRE}_{\mathrm{discr}}$ and corresponding iterations provided by the iteration (12) with $Z_{\text {circ }}, Z_{\text {struct }}$ and $Z_{\mathrm{ART}}$ preconditioners. We notice that in most cases (the only exceptions are the ones represented by $Z_{\text {struct }}$ for $\alpha=0.01,0.005$ ) the discrepancy principle does not work, probably because of the very low level of noise that characterizes the problem. As in Example 1 , the RREs relative to $Z_{\text {struct }}$ are smaller than all the other ones. However, unlike Example 1, the preconditioner built from the symmetrized version of the original PSF, i.e. $Z_{\mathrm{ART}}$ since we are in the Anti-Reflective framework, shows good performance, while $Z_{\text {circ }}$ gives rise to low quality restorations (especially for small values of $\alpha$ ). These facts can be visually appreciated by looking at the restored images reported in Figure 5.

As shown in Table 4, for the two sequences (geometric and DH) we have that $Z_{\text {struct }}^{k}, Z_{\text {circ }}^{k}$ and $Z_{\mathrm{ART}}^{k}$ exhibit a similar behaviour in terms of speed, however $Z_{\text {circ }}^{k}$ reaches a lower accuracy. We underscore that, except the DH strategy, in all the other cases the discrepancy principle fails. Even if CGLS is the slowest and the least accurate among the methods taken into account, its performance can be greatly improved by the use of a suitable structured preconditioner. Indeed PCGLS (with $\alpha=0.0004$ ) in this case is competitive with $Z_{\mathrm{struct}}^{k}$ and $Z_{\mathrm{ART}}^{k}$. The efficacy of these two strategies appears to be very similar. In particular, the first method is the best for the geometric sequence, while the second is the best for the 


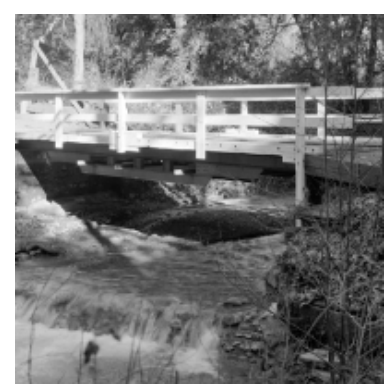

(a)

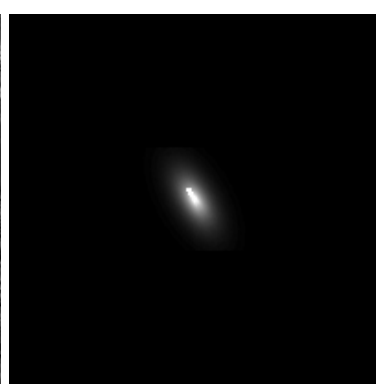

(b)

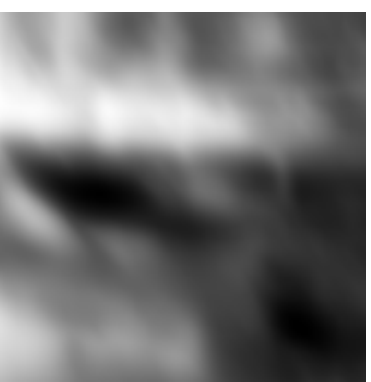

(c)

Figure 4: Example 2: (a) true image; (b) PSF; (c) blurred and noisy image.

$\mathrm{DH}$ one. About this sequence in relation to $Z_{\mathrm{ART}}^{k}$, we underline that in this example it is necessary to choose $\rho_{\mathrm{ART}}$ very carefully (in particular equal to $5 \cdot 10^{-2}$ ) in order to obtain an effective method; indeed, moving away from that value, you get soon a significant loss of accuracy. In general, in case of "almost" symmetric PSFs, we may observe that $Z_{\mathrm{ART}}^{k}\left(Z_{\mathrm{DCT}}^{k}\right.$ for problems with Reflective BCs) overcomes the proposed $Z_{\text {struct }}^{k}$ in terms of accuracy. Surprisingly enough, this seems to be caused just in small part by the different transforms involved (i.e. FFT for $Z_{\text {struct }}^{k}$, ART and DCT for $Z_{\mathrm{ART}}^{k}$ and $Z_{\mathrm{DCT}}^{k}$, respectively), while the main role seems to be played by the symmetrization process, which has a positive effect for PSFs close to symmetry (while it has a negative effect for strongly non-symmetric PSFs, as observed in Example 1). Therefore, in order to exploit this possibility, one may think to add in Algorithm 1 a symmetrization step that is on or off depending on the symmetry properties of the PSF at hand.

\begin{tabular}{|c|c|c||c|c||c|c||c|c|}
\cline { 2 - 9 } \multicolumn{1}{c|}{} & \multicolumn{2}{c||}{$\alpha=0.01$} & \multicolumn{2}{c||}{$\alpha=0.005$} & \multicolumn{2}{c||}{$\alpha=0.001$} & \multicolumn{2}{c|}{$\alpha=0.0005$} \\
\cline { 2 - 9 } \multicolumn{1}{c|}{} & $\mathrm{RRE}_{\min }$ & $\mathrm{RRE}_{\text {discr }}$ & $\mathrm{RRE}_{\min }$ & $\mathrm{RRE}_{\text {discr }}$ & $\mathrm{RRE}_{\min }$ & $\mathrm{RRE}_{\text {discr }}$ & $\mathrm{RRE}_{\min }$ & $\mathrm{RRE}_{\text {discr }}$ \\
\hline$Z_{\text {struct }}$ & $0.1808(61)$ & $0.1821(87)$ & $0.1808(31)$ & $0.1828(47)$ & $0.1811(6)$ & $-(-)$ & $0.1814(3)$ & $-(-)$ \\
\hline$Z_{\text {circ }}$ & $0.1917(40)$ & $-(-)$ & $0.2029(5)$ & $-(-)$ & $0.2041(1)$ & $-(-)$ & $0.2022(1)$ & $-(-)$ \\
\hline$Z_{\text {ART }}$ & $0.1856(58)$ & $-(-)$ & $0.1854(29)$ & $-(-)$ & $0.1842(6)$ & $-(-)$ & $0.1835(4)$ & $-(-)$ \\
\hline
\end{tabular}

Table 3: Example 2: $\mathrm{RRE}_{\min }$ and $\mathrm{RRE}_{\text {discr }}$ and corresponding iterations (in parentheses) for $Z_{\text {struct }}, Z_{\text {circ }}, Z_{\mathrm{ART}}$ preconditioners.

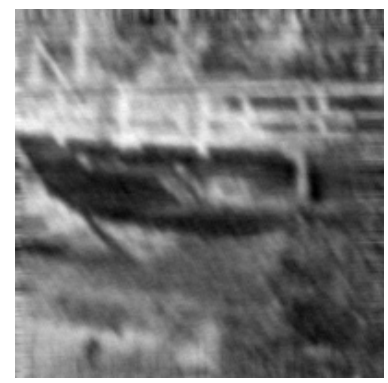

(a) $Z_{\text {struct }}, \alpha=0.005$

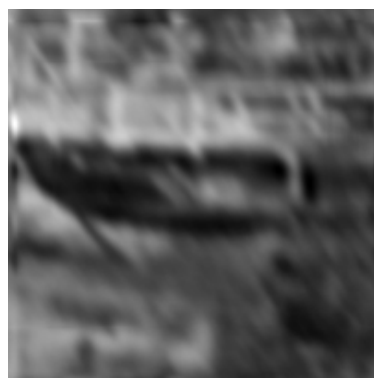

(b) $Z_{\text {circ }}, \alpha=0.005$

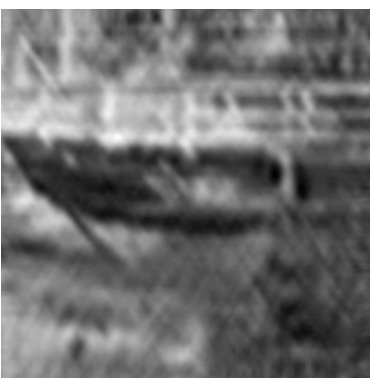

(c) $Z_{\mathrm{ART}}, \alpha=0.005$

Figure 5: Example 2: (a) Optimal reconstruction with $Z_{\text {struct }}$; (b) Optimal reconstruction with $Z_{\text {circ }}$; (c) Optimal reconstruction with $Z_{\mathrm{ART}}$.

\section{Conclusions}

In this paper we have considered iterative methods for image restoration and we have proposed a preconditioning technique which preserves the structure of the blurring matrix, which is determined by the BCs imposed in the problem. The presented preconditioning strategy represents a generalization and an improvement with respect of both circulant $[5,18]$ and structured preconditioning available in the literature $[11,9,10]$. Moreover the proposal has been further extended to provide a non-stationary preconditioning in the same spirit of a recent proposal for BCCB matrices [12]. In particular, the proposed new structured preconditioning gives rise to more accurate restorations and shows higher stability and robustness with respect to the BCCB approach since iterative methods compute good results even when the regularization parameters are very small. 


\begin{tabular}{|c|c|c|c|c|c|c|c|c|}
\hline & \multicolumn{3}{|c|}{ geometric } & \multicolumn{3}{|c|}{ DH } & \multirow{2}{*}{ CGLS } & \multirow{2}{*}{ PCGLS } \\
\hline & $Z_{\text {struct }}^{k}$ & $Z_{\text {circ }}^{k}$ & $Z_{\mathrm{ART}}^{k}$ & $Z_{\text {struct }}^{k}$ & $Z_{\text {circ }}^{k}$ & $Z_{\mathrm{ART}}^{k}$ & & \\
\hline $\mathrm{RRE}_{\min }$ & $0.1808(20)$ & 0.1919 (19) & $0.1845(20)$ & $\mathrm{n} / \mathrm{a}$ & $\mathrm{n} / \mathrm{a}$ & $\mathrm{n} / \mathrm{a}$ & 0.2014 (116) & $0.1851(24)$ \\
\hline $\mathrm{RRE}_{\text {discr }}$ & $-(-)$ & $-(-)$ & $-(-)$ & $0.1854(16)$ & $0.1940(12)$ & $0.1834(10)$ & $-(-)$ & $-(-)$ \\
\hline
\end{tabular}

Table 4: Example 2: $\mathrm{RRE}_{\mathrm{min}}, \mathrm{RRE}_{\mathrm{discr}}$ and corresponding iterations (in parentheses) for $Z_{\mathrm{struct}}^{k}, Z_{\text {circ }}^{k}, Z_{\mathrm{ART}}^{k}$ preconditioners for both the geometric and DH sequences and for CGLS and PCGLS. We fix $\rho_{\text {struct }}=10^{-2}, \rho_{\text {circ }}=10^{-1}$ and $\rho_{\text {ART }}=5 \cdot 10^{-2}$.

About future research lines, we mention that, in order to preserve edges or to enforce sparsity in a certain bases (usually wavelet) on the restored image, regularization terms that lead to non-linear problems are usually employed. Nevertheless, the resulting numerical methods usually require the solution of a regularized least square method, e.g. the linearized Bregman splitting [20, 21]. Therefore, improvements in classical least square methods can be useful also for these more computationally challenging models as shown in [22] where among other strategies the reblurring preconditioner and its non-stationary variants introduced in [5] and [12], respectively, have been adapted to be included in the linearized Bregman splitting for the synthesis approach proposed in [20]. The structure preserving preconditioners proposed in this paper could be similarly considered to improve such numerical methods. This will be subject of further studies.

\section{Acknowledgements}

This work is partly supported by PRIN 2012 N. 2012MTE38N and GNCS-INdAM.

\section{References}

[1] P. C. Hansen, Rank-deficient and discrete ill-posed problems, SIAM Publications, Philadelphia, PA .

[2] P. C. Hansen, J. Nagy, D. P. O’Leary, Deblurring Images Matrices, Spectra and Filtering, SIAM Publications, Philadelphia, PA

[3] A. Aricò, M. Donatelli, S. Serra-Capizzano, Spectral analysis of the anti-reflective algebra, Linear Algebra and its Applications 428 (2008) 657-675.

[4] M. Piana, M. Bertero, Projected Landweber method and preconditioning, Inverse Problems 13 (2) (1997) $441-463$.

[5] P. Dell' Acqua, M. Donatelli, C. Estatico, Preconditioners for image restoration by reblurring techniques, Journal of Computational and Applied Mathematics 272 (2014) 313-333.

[6] M. Donatelli, S. Serra-Capizzano, Anti-reflective boundary conditions and re-blurring, Inverse Problems 21 (1) (2005) 169-182.

[7] S. Serra-Capizzano, E. Tyrtyshnikov, Any Circulant-Like Preconditioner for Multilevel Matrices Is Not Superlinear, SIAM Journal on Matrix Analysis and Applications 21 (2) (1999) 431-439.

[8] S. Serra-Capizzano, Preconditioning strategies for asymptotically ill-conditioned block Toeplitz systems, BIT Numerical Mathematics 34 (4) (1994) 579-594.

[9] M. Hanke, J. Nagy, Inverse Toeplitz preconditioners for ill-posed problems, Linear Algebra and its Applications 284 (1-3) (1998) 137-156, international Linear Algebra Society (ILAS) Symposium on Fast Algorithms for Control, Signals and Image Processing.

[10] M. K. Ng, R. H. Chan, W. C. Tang, A Fast Algorithm for Deblurring Models with Neumann Boundary Conditions, SIAM Journal on Scientific Computing 21 (3) (1999) 851-866.

[11] P. Dell'Acqua, M. Donatelli, S. Serra-Capizzano, D. Sesana, C. T. Possio, Optimal preconditioning for image deblurring with Anti-Reflective boundary conditions, Linear Algebra and its Applications In press.

[12] M. Donatelli, M. Hanke, Fast nonstationary preconditioned iterative methods for ill-posed problems, with application to image deblurring, Inverse Problems 29 (2013) 095008, doi:10.1088/0266-5611/29/9/095008.

[13] J. M. Chung, M. E. Kilmer, D. P. O’Leary, A Framework for Regularization via Operator Approximation, SIAM Journal on Scientific Computing 37 (2) (2015) B332-B359.

[14] M. Donatelli, Fast transforms for high order boundary conditions in deconvolution problems, BIT Numerical Mathematics 50 (3) (2010) $559-576$.

[15] S. Serra-Capizzano, A note on anti-reflective boundary conditions and fast deblurring models, SIAM Journal on Scientific Computing 25 (3) (2003) 1307-1325.

[16] M. Donatelli, S. Serra-Capizzano, Antireflective Boundary Conditions for Deblurring Problems, Journal of Electrical and Computer Engineering (2010) 1-18Article ID 241467.

[17] C. Estatico, A classification scheme for regularizing preconditioners, with application to Toeplitz systems, Linear Algebra and its Applications 397 (2005) 107-131.

[18] M. Hanke, J. Nagy, R. Plemmons, Preconditioned iterative regularization for ill-posed problems, Numerical Linear Algebra (1993) 141163Proceedings of the Conference in Numerical Linear Algebra and Scientific Computation,Kent, Ohio, March 13-14 1992.

[19] R. M. Gray, Toeplitz and Circulant Matrices: A Review, Foundations and Trends in Communications and Information Theory 2 (3) (2006) 155239.

[20] X. Liu, L. Huang, Split Bregman iteration algorithm for total bounded variation regularization based image deblurring, Journal of Mathematical Analysis and Applications 372 (2) (2010) 486-495.

[21] J. Huang, M. Donatelli, R. H. Chan, Nonstationary iterated thresholding algorithms for image deblurring, Inverse Problems and Imaging 7 (3) (2013) 717-736.

[22] Y. Cai, D. Bianchi, M. Donatelli, T. Z. Huang, Regularization preconditioners for frame-based image deblurring with reduced boundary artifacts, SIAM Journal on Scientific Computing In press. 
